\title{
Sex chromosome positions in human interphase nuclei as studied by in situ hybridization with chromosome specific DNA probes
}

\author{
G.A. Rappold ${ }^{1}$, T. Cremer ${ }^{1}$, H.D.Hager ${ }^{1}$, K.E.Davies ${ }^{2}$, C.R.Müller ${ }^{3}$, and T. Yang ${ }^{4}$ \\ ${ }^{1}$ Institut für Anthropologie und Humangenetik, Universität Heidelberg, Im Neuenheimer Feld 328, D-6900 Heidelberg, \\ Federal Republic of Germany \\ ${ }^{2}$ Biochemistry Department, St. Mary's Hospital Medical School, University of London, Paddington, London W21PC, England \\ ${ }^{3}$ Institut für Humangenetik, Universität Würzburg, Koellikerstr. 2, D-8700 Würzburg \\ ${ }^{4}$ Institute of Human Genetics, Texas Medical Center, Houston, TX 77030, USA
}

\begin{abstract}
Summary. Two cloned repetitive DNA probes, pXBR and CY1, which bind preferentially to specific regions of the human $\mathrm{X}$ and $\mathrm{Y}$ chromosome, respectively, were used to study the distribution of the sex chromosomes in human lymphocyte nuclei by in situ hybridization experiments. Our data indicate a large variability of the distances between the sex chromosomes in male and female interphase nuclei. However, the mean distance observed between the $\mathrm{X}$ and $\mathrm{Y}$ chromosome was significantly smaller than the mean distance observed between the two X-chromosomes. The distribution of distances determined experimentally is compared with three model distributions of distances, and the question of a non-random distribution of sex chromosomes is discussed. Mathematical details of these model distributions are provided in an Appendix to this paper. In the case of a human translocation chromosome (Xqter $\rightarrow$

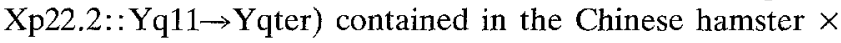
human hybrid cell line $445 \times 393$, the binding sites of pXBR and CY1 were found close to each other in most interphase nuclei. These data demonstrate the potential use of chromosome-specific repetitive DNA probes to study the problem of interphase chromosome topography.
\end{abstract}

\section{Introduction}

Evidence that the arrangement of metaphase chromosomes does not occur at random has been obtained for a number of plant species and to a minor degree also for mammals including man (Bennett 1983; Comings 1980). In addition, it has been shown that such arrangements to a certain extent still reflect the arrangement of chromosome territories (also called chromosome domains) in the interphase nucleus (Cremer et al. 1984). In spite of this progress, the problem of chromosome topography in interphase nuclei and its possible functional significance (Lewin 1981; Vogel and Krüger 1983) have remained matters of great controversy and uncertainty (for reviews see Comings 1980; Avivi and Feldman 1980).

Offprint requests to: Dr. T. Cremer, Institut für Anthropologie und Humangenetik, Universität Heidelberg, Im Neuenheimer Feld 328, D-6900 Heidelberg, Federal Republic of Germany
Use of metaphase plates as a substitute for direct studies of interphase chromosome arrangements has two major disadvantages:

1. The extent to which chromosome arrangements at metaphase actually reflect their relative positions in the preceding interphase nucleus depends on numerous factors which are difficult to control. Such factors include chromosome condensation, natural movements of mitotic chromosomes and artifacts introduced by the various methods of preparation and evaluation of metaphase plates.

2. Studies of metaphase plates are principally limited to cycling cells and have to rely on the assumption that the evaluated sample of mitotic cells is representative for the whole cell population. This assumption seems especially questionable for cells of tissues in vivo, in which cell cycle transit times vary greatly in different types of differentiated cells, including terminally differentiated cells which do not cycle at all.

To overcome the first of the two disadvantages mentioned above, two approaches have been established in our institute. One approach makes use of a laser-UV-microbeam, which allows microirradiation of small parts of interphase nuclei. Microirradiated chromatin can be followed from interphase to metaphase and its distribution on metaphase chromosomes can be studied (Cremer et al. 1982 a, b; Hens et al. 1983). The other approach is based on the analysis of chromosome exchange patterns which occur either spontaneously in lymphocytes from patients with Fanconi's anemia and Bloom's syndrome or can be induced experimentally in human lymphocytes or in Chinese hamster cells by mitomycin C or X-rays (Hager et al. 1982; Teltschik and Schroeder, unpublished data). The first approach is based on the assumption that the probability of microirradiated cells entering the first postirradiation mitosis is similar for all possible combinations of chromosomes damaged at the microirradiated unclear sites. The second approach is based on the assumption that the frequency of exchanges between individual chromosomes is closely correlated with their proximity during interphase. The validity of these assumptions is difficult to prove. In particular, neither approach overcomes the second disadvantage involved in studies of metaphase chromosome arrangements. For this purpose we urgently need methods which allow the direct visualization of individual chromosomes or parts thereof in interphase 
nuclei. Such methods are presently limited to a few heterochromatic chromosome regions which can be stained distinctly both in interphase and metaphase. For example, the positions of chromosomes No.9 in human interphase nuclei can be observed by means of the Giemsa-11-staining procedure (Gagné and Laberge 1972; Spaeter 1975). Another example concerns the position of the heterochromatic sex chromosomes forming large chromocenters in interphase nuclei of Microtus agrestis (Pera and Schwarzacher 1970), while the position of the human Y-heterochromatin can be identified by quinacrine mustard staining (Pearson et al. 1970; Schmid et al. 1981).

In this paper we describe a new approach by which we have visualized the positions of both sex chromosomes in male and female interphase nuclei of human lymphocytes simultaneously. This approach makes use of two cloned DNA probes CY1 (Müller et al. 1983) and pXBR (Yang et al. 1982) which hybridize in situ predominantly to the human $\mathrm{Y}$ and $\mathrm{X}$ chromosome, respectively. Distances between the two X-chromosomes in female nuclei and between the $\mathrm{X}$ and the Y-chromosome in male nuclei were determined and compared with each other and with three model distributions of distances, calculated under the assumption that two points (i.e., the labelled regions of the two sex chromosomes) were distributed independently from each other within a sphere (i.e., the human lymphocyte nucleus). Mathematical details of these models are described by Klar et al. in an Appendix to this paper. For comparison we have used a Chinese hamster $x$ human cell line containing a human translocation chromosome (Xqter $\rightarrow$ Xp22.2: $:$ Yq11 $\rightarrow$ Yqter) to study the distribution of the in situ hybridization sites of the two DNA probes in nuclei where the two sites are physically linked on one chromosome.

\section{Materials and methods}

Molecular probes. A tandemly repeated DNA sequence, pXBR, organized predominantly at or near the centromeric region of the human $\mathrm{X}$ chromosome, was used (Yang et al. 1982). Female placental DNA was used as a source of DNA for cloning. After digestion with $\mathrm{Bam} \mathrm{H} 1$ and fractionation on preparative agarose gels, a $2 \mathrm{~Kb}$ band was recovered from the gel, ligated to Bam H1-digested pBR 322 DNA and transfected into Escherischia coli HMS 175.

Clone CY1 was derived by Benton-Davis plaque screening from a Y chromosome library (Müller et al. 1983; Davies, unpublished). It consists of two 3.4 and $2.6 \mathrm{~Kb}$ long Eco R1 inserts cloned in a $\lambda$ gtWES $\lambda \mathrm{B}$ vector. With in situ hybridization, about $80 \%$ of all grains observed on metaphase plates were located exclusively on $\mathrm{Yq}$ with a second minor binding site in the centromeric region of chromosome 9. Thus CY1 represents a repetitive sequence which hybridizes predominantly to the $\mathrm{Y}$ chromosome. For in situ hybridization the two DNA probes were labelled to a specific activity of $2 \times 10^{7} \mathrm{cpm} / \mu \mathrm{g}$ DNA by nick translation (essentially according to Kunkel et al. 1979) with (3H)dTTP (100 Ci/mmol; New England Nuclear Corp.; $1 \mathrm{Ci}=3.7 \times 10^{10}$ becquerels $)$.

Cell cultures. For in situ hybridization experiments we used phytohaemagglutinin-stimulated human peripheral blood lymphocyte cultures $(72 \mathrm{~h})$ and a Chinese hamster $\times$ human cell line $445 \times 393$ which contains a human translocation chromosome $(X q t e r \rightarrow X p 22.2::$ Yq11 $\rightarrow$ Yqter) and no normal $\mathrm{X}$ or $\mathrm{Y}$ chromosome. This cell line was kindly supplied by Dr. H.H. Ropers and described in detail by Goodfellow et al. (1983). Cells were grown under standard conditions (minimum essential medium, $10 \%$ fetal calf serum, $5 \% \mathrm{CO}_{2}$ ) in monolayer culture. Lymphocyte cultures were derived from probands with normal karyotypes 46, XY and 46,XX.

In situ hybridization. Slides were precoated by incubation in Denhardt's solution at $65^{\circ} \mathrm{C}$ (Gerhard et al. 1981), washed in water and air dried. Mitotic cells were arrested by colcemid $(0.1 \mu \mathrm{g} / \mathrm{ml})$, treated by hypotonic shock $(0.075 \mathrm{M} \mathrm{KCl}, 20-$ $30 \mathrm{~min})$, fixed in methanol/acetic acid $(3: 1)$ and spread on the precoated slides. Chromosome preparations were used within

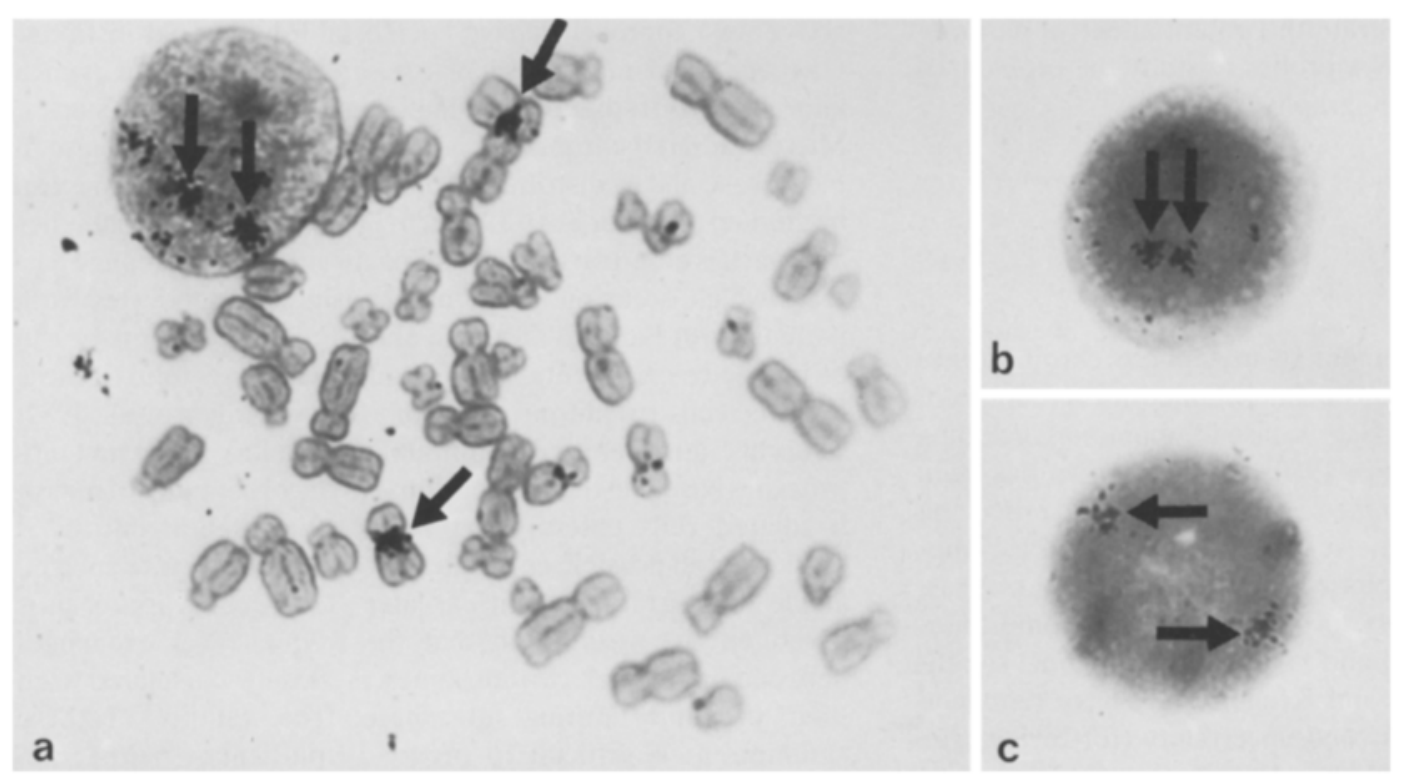

Fig.1a-c. In situ hybridization of clone pXBR to female human lymphocytes. a Metaphase plate stained with Giemsa showing strong hybridization signals at the centromeric regions of the two X-chromosomes (arrows). Two major hybridization sites were also observed in interphase nuclei (arrows; a, upper left, $\mathbf{b}$ and $\mathbf{c}$ ). The three nuclei demonstrate the large variation of the distances between Xcen in interphase nuclei of human lymphocytes 
3 weeks. In situ hybridization was carried out as described in detail by Rappold and Vosberg (1983) and Rappold et al. (1984). Briefly, denaturation of chromosomal DNA was performed with $70 \%$ formamide $/ 30 \% 2 \times \mathrm{SSC}$ at $70^{\circ} \mathrm{C}$ for $2 \mathrm{~min}$, followed by dehydration in ethanol. Denaturation of the $\left({ }^{3} \mathrm{H}\right)$ labeled probe DNA was carried out at $100^{\circ} \mathrm{C}$ for $5 \mathrm{~min}$. Hybridization of the probe under annealing conditions was carried out for $16 \mathrm{~h}$ at $40^{\circ} \mathrm{C}$. After extensive washings in $2 \times \mathrm{SSC}$ at $65^{\circ} \mathrm{C}$ and then in $0.1 \times \mathrm{SSC}$ at room temperature, slides were dehydrated in an ethanol series and air dried. Slides were coated with Ilford L4 emulsion (1:1) and exposed up to 1 week. Exposed slides were developed in Ilford JD 19 developer. Autoradiographs were stained with Giemsa (Gurr) or quinacrin mustard (Sigma) and photographed using a Zeiss photomicroscope equipped with epifluorescence illumination.

Evaluation of cell nuclei. Nuclei showing two major binding sites following in situ hybridization of female lymphocytes with pXBR or of male lymphocytes and $445 \times 393$ cells respectively with the mixed probes CY1 and $\mathrm{pXBR}$ were evaluated from enlarged microphotographs. The distance between the centers of the two binding sites, i.e., spots of accumulated silver grains, was measured and divided by the diameter of the nucleus. In the case of non-circular nuclei the horizontal diameter was arbitrarily determined. The resulting normalized distances $\alpha$ between the two binding sites obtained in each sample of evaluated nuclei were grouped in 20 classes ranging from $0-0.05$ to $0.95-1.0$. The observed normalized mean distances were compared with their expectation derived from three model distributions (see Results and Appendix).

\section{Results}

In situ hybridization of cloned DNA-probes CY1 and $p X B R$ to male and female lymphocytes. Figure 1 shows examples of in situ hybridization experiments of clone $\mathrm{pXBR}$ to lymphocytes of two healthy females. Two major sites of binding were observed both in metaphase spreads over the centromeric regions of the two $\mathrm{X}$ chromosomes and over interphase nuclei indicating the sites of the centromeric regions of the two $\mathrm{X}$ chromosomes in interphase nuclei. Under the conditions used in the present experiment there was only minor hybridization
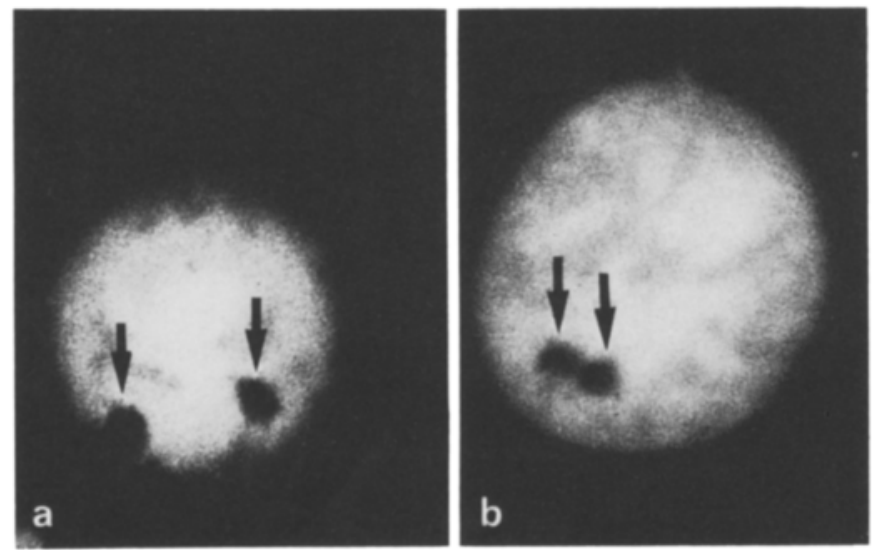

Fig. 2 a and b. In situ hybridization with mixed probes $\mathrm{pXBR}$ and $\mathrm{CY} 1$ to male human lymphocytes. a, b Microphotographs of two nuclei stained with quinacrine mustard. Fluorescence was spared at the hybridization sites (arrows) as a result of silver grain accumulation over these sites which interfered with epifluorescence illumination of $\mathrm{pXBR}$ and $\mathrm{CY} 1$ respectively to autosomal sites. Interphase nuclei where the distinction between minor hybridization sites (to autosomes) and major hybridization (to the sex chromosomes) was not clearly possible were excluded from further evaluation. In contrast, in situ hybridization of either pXBR or CY1 alone to male human lymphocyte nuclei generally resulted in an accumulation of silver grains at one major binding site only, representing the position of the labelled parts of either $\mathrm{X}$ or $\mathrm{Y}$ chromosome. Two distinctly separated spots were rarely seen in these experiments $(1 \%)$.

In the case of in situ hybridization experiments of the mixed probes pXBR and CY1 to human lymphocytes of two healthy males, both the long arm of the $Y$ chromosome and the centromeric region of the $\mathrm{X}$ chromosome appeared heavily labelled in metaphase spreads. Accordingly, interphase nuclei showed two major sites of hybridization indicating the relative positions

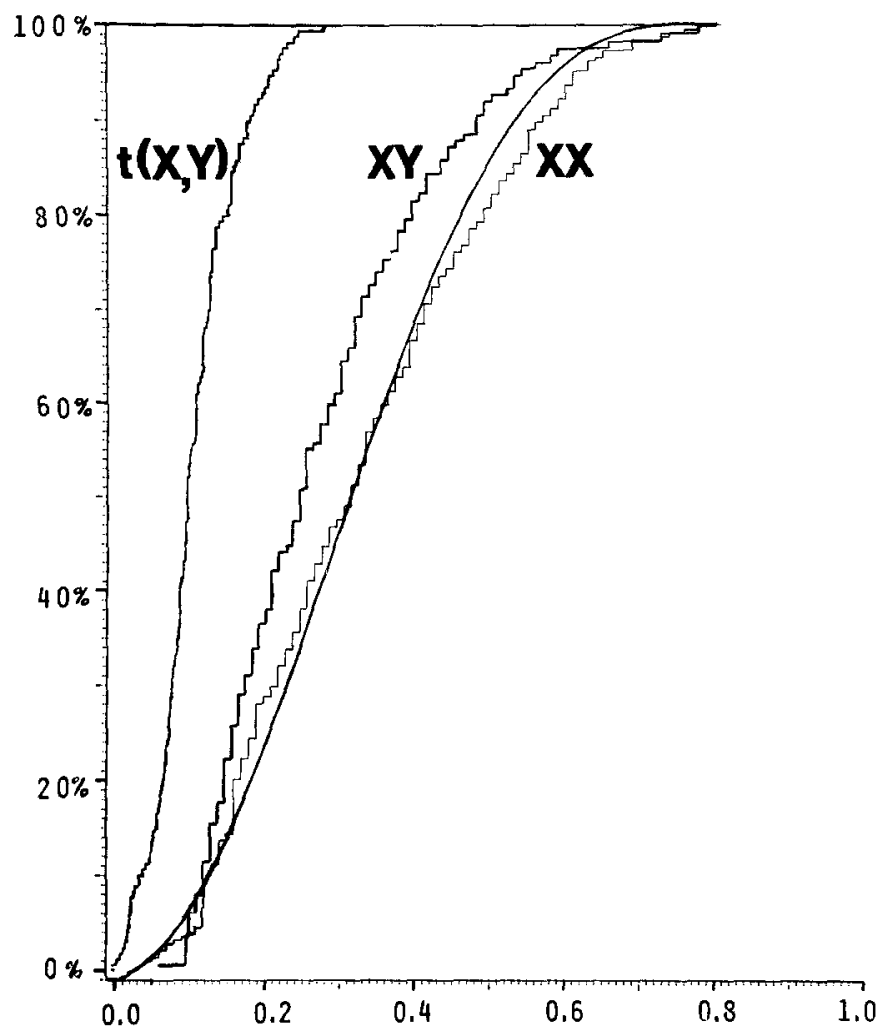

Fig.3. Abscissa: Normalized distances $\beta$ between two major binding sites (see Material and methods). Ordinate: Cumulative fraction of nuclei showing a normalized distance $\beta$ equal to or smaller than the corresponding $\beta$ on the abscissa. $X X$ : Cumulative fraction of $\beta$ derived for the two binding sites of $\mathrm{pXBR}$ in female human lymphocyte nuclei $(n=280)$. Data were pooled from two independent cases which gave a very similar distribution of distances. $X Y$ : Cumulative fraction of $\beta$ obtained for the two binding sites of pXBR and CY1 in male human lymphocyte nuclei $(n=399)$. Data were pooled from two independent cases showing a very similar distribution of distances. $t(X ; Y)$ : Cumulative fraction of $\beta$ obtained for the two binding sites of $\mathrm{pXBR}$ and CY1 in nuclei $(n=245)$ of $445 \times 393$ hybrid cells indicating the normalized distances between the centromeric region of the human $X$ and the translocated part of the short arm of the human $Y$ in the translocation chromosome (Xqter $\rightarrow \mathrm{Xp} 22.2:: \mathrm{Yq} 11 \rightarrow \mathrm{Yqter}$ ). The smooth curve shows the cumulative fraction of $\beta$ obtained in the case of model a described in detail in the Appendix [compare equation 11: $G_{2,0}(\mathrm{~b})$ ]. Two assumptions were made: (i) The lymphocyte nucleus is a sphere. (ii) Two points indicating the positions of the Iabelled chromosome segments are distributed uniformly and independently from each other within the sphere. (For further details see Results and Appendix) 


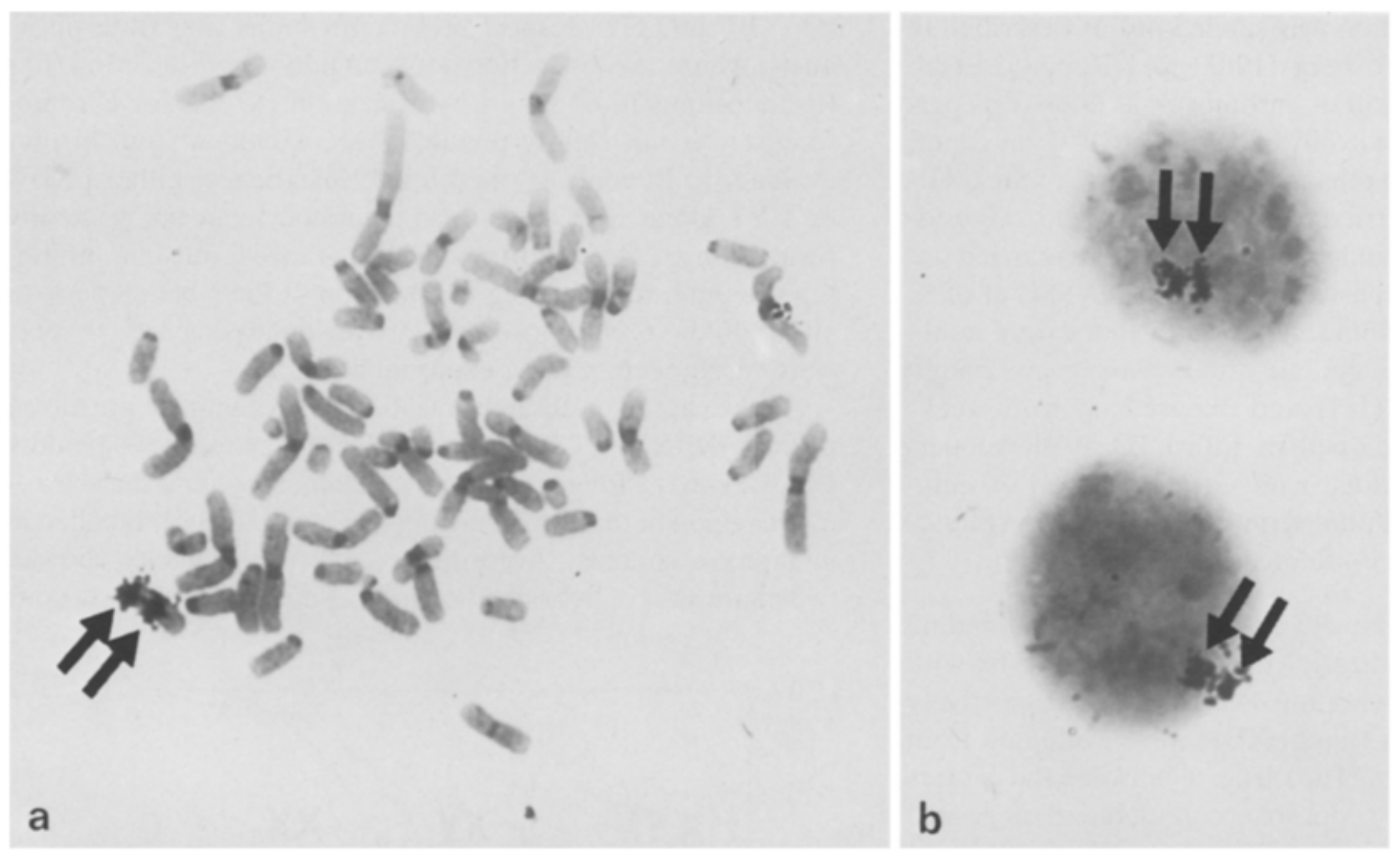

Fig.4a and b. In situ hybridization with mixed probes pXBR and CY1 to a metaphase spread (a) and two interphase nuclei (b) of the Chinese hamster $\times$ human cell hybrid line $445 \times 393$, containing a human translocation chromosome $(\mathrm{Xqter} \rightarrow \mathrm{Xp} 22.2::$ Yq11 $\rightarrow$ Yqter). In this chromosome the centromeric region of the $\mathrm{X}$ and the translocated part of $\mathrm{Yq}$ could be simultaneously labelled with the two probes (a, arrows). The distances between the two labelled sites observed in interphase nuclei reflect the extent of elongation of the investigated chromosome arm during interphase $(\mathbf{b}$, arrows $)$

of the labelled parts of the $\mathrm{X}$ and $\mathrm{Y}$ chromosomes. When interphase nuclei were counterstained with quinacrine mustard, fluorescence could not be observed at the hybridization sites due to the accumulation of silver grains over these sites, which interferes with epifluorescence illumination (Fig.2).

For quantitative evaluation, samples of interphase nuclei showing two clear sites of hybridization were randomly taken. Normalized distances $\alpha$ (see Materials and methods) determined between $X, X$ and $X, Y$ positions in female and male human lymphocyte nuclei are shown in Fig. 3. For comparison Fig. 3 shows a model curve of distances [see Appendix; model a, equation (11)] obtained under the assumptions that (i) the human lymphocyte interphase nucleus has a spherical shape and (ii) two points indicating the positions of the respective chromosome segments are distributed uniformly and independently of each other within the sphere. Distances between each possible combination of two points were calculated after orthogonal projection onto a plane. Due to the method of evaluation, the maximum experimental distance was necessarily somewhat smaller than 1 even in cases where the positions of the two labelled sites were exactly opposite each other at the nuclear equator. Accordingly, the diameter of the model sphere was assumed to be equal to the maximum normalized distance $\beta_{\max }$ determined experimentally. This was done in order to minimize a possible difference between the distribution of distances predicted by the model and the experimental distances. For $\alpha_{\max }=0.85$ the mean distance $\bar{\alpha}$ obtained for the model curve was 0.34 as compared to $\bar{\alpha}=0.40$ in case of $\alpha_{\max }=1.0$ [see Appendix; model a, equation (12c)]. When compared with this model curve, cumulative distances obtained from lymphocyte nuclei of the two male individuals still showed a significantly smaller mean distance of the labelled $\mathrm{X}$ and $Y$ chromosome segments $(0.30)$. In contrast, no significant difference between this model curve of distances and the experimental curve of distances was obtained for the $\mathrm{X}$ chromosomes in lymphocyte nuclei of the female individuals ( 0.34 observed as compared to 0.33 expected) (Fig. 3). In addition to model a, two other model distributions were also considered (see Discussion and Appendix, models $\mathbf{b}$ and $\mathrm{c}$ ). These models take into account the possibility that the labeled region of one or both sex chromosomes is preferentially situated at the nuclear periphery, and notably predict a larger mean distance between the labeled segments as compared to model a. Accordingly, the mean distances obtained experimentally are significantly smaller than predicted by models $b$ and $c$ not only for the sex chromosomes in male but also in female nuclei.

In situ hybridization of $C Y 1$ and $p X B R$ to the Chinese hamster $\times$ human hybrid cell $445 \times 393$. In situ hybridization experiments $(n=4)$ with the mixed probes PXBR and CY1 were also performed in the Chinese hamster $\times$ human cell hybrid line $445 \times 393$ containing a human translocation chromosome

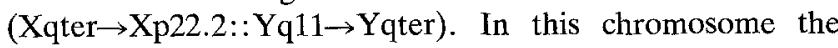
centromeric region of the $\mathrm{X}$ and the translocated part of $\mathrm{Yq}$ could be simultaneously labelled by hybridization with the two probes (Fig. 4). The distances between the two labelled sites as observed in interphase nuclei then reflect the extent of elongation of the investigated chromosome arm during interphase. In most cases the major binding sites of the two probes were found in close proximity (Figs. 3, 4), suggesting that this chromosome arm was generally retained within a rather compact nuclear domain.

\section{Discussion}

Two cloned DNA probes, $p X B R$ and CY1, which bind to specific regions of the human $\mathrm{X}$ and $\mathrm{Y}$ chromosome, were used in the present investigation to study the relative positions of the 
two sex chromosomes directly within interphase nuclei of female and male human lymphocytes. Data obtained for two normal female and two normal male individuals show a large variability of the distances between the two sex chromosomes in interphase nuclei (Fig. 3). Yet our data do not exclude the possibility that each sex chromosome might occur at a preferential position in a haploid set or subset of chromosomes. In such a case, however, these data indicate that the relative arrangements of these ordered sets would vary largely from cell to cell.

For several plant species evidence has been presented for a highly ordered arrangement of chromosomes in haploid sets as predicted by a model proposed by Bennett (1983). If an ordered arrangement of haploid chromosome sets exists in mammalian interphase nuclei, one can predict differences in the distribution of distances between different pairs of non-homologous chromosomes. In situ hybridization with mixed probes of chromosome specific repetitive DNAs obtained from different chromosomes, when compared with the in situ hybridization pattern of each probe alone, could be used to study this question. Interestingly, the mean distance between the $X$ and the $\mathrm{Y}$ chromosome was significantly smaller than the mean distance between the two $\mathrm{X}$ chromosomes. The feasibility of this method is further demonstrated by the fact that the mean distance between the hybridization sites of CY1 and pXBR was very small in interphase nuclei of the Chinese hamster $x$ human cell line $445 \times 393$. At present such investigations are limited by the number of probes available (Gosden et al. 1981; Law et al. 1982; Kanda et al. 1982).

The choice of the model distribution of distances used for comparison with the experimentally measured distances causes particular problems (see Appendix). The distribution of distances between the two major binding sites of $\mathrm{pXBR}$ in female human lymphocyte nuclei closely follows the distribution predicted by model a (see Appendix). In this case the centromeres of the two $\mathrm{X}$-chromosomes are considered to be two points distributed uniformly and independently from each other within a sphere (Fig. 3). Since it is well established that the inactivated $X$ in many nuclei is located at the nuclear periphery, model $\mathrm{b}$ in the Appendix considers one point to be distributed uniformly at the surface of a sphere, while the other point is distributed within the sphere. A third model $\mathrm{c}$ considers both points to be distributed at the surface of the sphere in a random manner. Notably, the mean distances $\bar{\alpha}$ between the two points obtained for model $\mathrm{b}[\bar{\alpha}=0.47$; see Appendix, equation (15c) $]$ and model c $[\bar{\alpha}=0.52$; see Appendix, equation (19c)] were considerably larger than $\bar{\alpha}$ for model a $[0.40$; see Appendix, equation (12c)]. In comparison to models $b$ and $c$ the experimentally determined distances between the centromeric regions of the two X-chromosomes were significantly smaller than the model distances. In other words, if the centromeric regions of one $\mathrm{X}$ or both $\mathrm{X}$-chromosomes in female nuclei were indeed located close to or at the nuclear periphery, the experimental data would suggest a distribution significantly closer than expected at random. The mean distance obtained between the $\mathrm{X}$ and $\mathrm{Y}$ chromosomes in male human lymphocyte nuclei was significantly smaller than the mean distances obtained for all three model distributions.

Difficulties in choosing an adequate model distribution of distances can in part be eliminated by a direct comparison of distances between different pairs of chromosomes in the interphase nucleus. Independent of the chosen model, significant differences between the mean distances of different pairs of chromosomes would indicate a non-random pattern of chromosome distribution.

A possible interpretation of the difference found between the distribution of the sex chromosomes in male and female nuclei could be that the small $Y$ chromosome was located preferentially at a more central region of the investigated nuclei, while the larger $\mathrm{X}$ chromosomes were generally distributed closer to the nuclear periphery (Hens et al. 1982). Further information on the three-dimensional position of the sex chromosomes within interphase nuclei is necessary to prove or disprove such an idea. In situ hybbridization of probes pXBR and CY1 to serially sectioned nuclei might provide such information.

Our data provide the first example of the potential use of cloned DNA probes to study the problem of interphase chromosome arrangements. It is a particular advantage of this approach that it can be extended to studies of interphase chromosome arrangements of cycling and non-cycling cells in vivo. Further studies are intended to clarify two questions: (i) Do other somatic cell types show the same variability in the arrangement of sex chromosomes as found in human lymphocytes? (ii) Does the arrangement of sex chromosomes in premeiotic stages of germ line cells differ from that found in somatic cell types?

\section{References}

Avivi L, Feldman M (1980) Arrangement of chromosomes in the interphase nucleus of plants. Hum Genet 55:281-295

Bennett MD (1983) The spatial distribution of chromosomes. In: Brandham PE, Bennett MD (eds) Kew Chromosome Conference II. George Allen \& Unwin, London, pp 71-79

Comings DE (1980) Arrangement of chromatin in the interphase nucleus. Hum Genet 53:131-143

Cremer T, Cremer C, Baumann H, Luedtke EK, Sperling K, Teuber V, Zorn C (1982a) Rabl's model of the interphase chromosome arrangement tested in Chinese hamster cells by premature chromosome condensation and laser-UV-microbeam experiments. Hum Genet 60:46-56

Cremer T, Cremer C, Scheider T, Baumann H, Hens L, KirschVolders M (1982 b) Analysis of chromosome positions in the interphase nucleus of Chinese hamster cells by laser-UV-microirradiation experiments. Hum Genet 62:201-209

Cremer T, Baumann H, Nakanishi K, Cremer C (1984) Correlation between interphase and metaphase chromosome arrangements as studied by laser-UV-microbeam experiments. Chromosomes Today, vol 8 (in press)

Gagné R, Laberge C (1972) Specific cytological recognition of the heterochromatic segment of number 9 chromosome in man. Exp Cell Res 73:239-242

Gerhard PS, Kawasaki ES, Bancroft FC, Szabo P (1981) Localization of an unique gene by direct hybridization in situ. Proc Natl Acad Sci USA 78:3755-3760

Goodfellow P, Banting G, Sheer D, Ropers HH, Caine A, FergusonSmith MA, Povey S, Voss R (1983) Genetic evidence for a Y-linked gene in humans homologous to a gene on the X-chromosome. Nature 302:346-349

Gosden JR, Lawrie SS, Cooke HJ (1981) A cloned repeated DNA sequence in human chromosome heteromorphisms. Cytogenet Cell Genet 29:32-39

Hager H-D, Schroeder-Kurth TM, Vogel F (1982) Position of chromosomes in the human interphase nucleus. An analysis of nonhomologous chromatid translocation in lymphocyte cultures after Trenimon treatment and from patients with Fanconi's anemia and Bloom's syndrome. Hum Genet 61:342-356 
Hens L, Kirsch-Volders M, Verschaeve L, Susanne C (1982) The central localization of the small and early replicating chromosomes in human diploid metaphase figures. Hum Genet 60:249-256

Hens L, Baumann H, Cremer T, Sutter A, Cornelis JJ, Cremer C (1983) Immunocytochemical localization of chromatin regions UVmicroirradiated in S-phase or anaphase. Evidence for a territorial organization of chromosomes during cell cycle of cultured Chinese hamster cells. Exp Cell Res 149:257-269

Kanda V, Schreck R, Alt F, Bruns G, Baltimore D, Latt S (1983) Isolation of amplified DNA sequences from IMR-32 human neuroblastoma cells. Facilitation by fluorescence-activated flow sorting of metaphase chromosomes. Proc Natl Acad Sci USA 80:40694073

Kunkel LM, Smith KD, Boyer SH (1979) Organization and heterogeneity of sequences within a repeating unit of human Y chromosome deoxyribonucleic acid. Biochemistry 18:3343-3353

Law ML, Davidson JN, Kao FT (1982) Isolation of a human repetitive sequence and its application to regional chromosome mapping. Proc Natl Acad Sci USA 79: 7390-7394

Lewin R (1981) Do chromosomes cross talk. Science 214: 1334-1335

Müller CR, Davies KE, Cremer C, Rappold G, Gray JW, Ropers HH (1983) Cloning of genomic sequences from the human $Y$ chromosome after purification by dual beam flow sorting. Hum Genet 64 : $110-115$

Pearson PL, Bobrow M, Vosa CG (1970) Technique for identifying Y chromosomes in human interphase nuclei. Nature 226:78-80
Pera F, Schwarzacher HG (1970) Localization of the heterochromatic chromosomes of Microtus agrestis in interphase and mitosis. Cytobiology 2: 188-199

Rappold GA, Vosberg H-P (1983) Chromosomal localization of a human myosin heavy-chain gene by in situ hybridization. Hum Genet $65: 195-197$

Rappold GA, Cremer T, Cremer C, Back W, Bogenberger J, Cooke HJ (1984) Chromosome assignment of two cloned DNA probes hybridizing predominantly to human sex chromosomes. Hum Genet 65:257-261

Schmid M, Poppen A, Schmid W, Engel W (1981) Somatic pairings of the $Y$ heterochromatin in human XYY and XY qi cells. Cytogenet Cell Genet 29:203-214

Spaeter M (1975) Nichtzufällige Verteilung homologer Chromosomen (Nr. 9 und $\mathrm{YY}$ ) in Interphasekernen menschlicher Fibroblasten. Hum Genet $27: 111-118$

Vogel F, Krüger J (1983) Is there a general relationship between estimated chromosome distances in interphase and location of genes with related functions? Hum Genet $63: 362-368$

Yang T, Hansen S, Oishi K, Ryder O, Hamkalo B (1982) Characterization of a cloned repetitious DNA sequence concentrated on the human X chromosome. PNAS 79: 6593-6597

Received March 28 / Revised April 10, 1984

\title{
Appendix
}

\section{Models for the distribution of distance of specific chromosome regions in interphase nuclei}

\author{
A. Klar ${ }^{1}$, J. Aichelin ${ }^{1}$, and J. Krüger ${ }^{2}$ \\ ${ }^{1}$ Max-Planck-Institut für Kernphysik, D-6900 Heidelberg \\ ${ }^{2}$ Institut für Anthropologie und Humangenetik, University of Heidelberg, Im Neuenheimer Feld 328, D-6900 Heidelberg, \\ Federal Republic of Germany
}

\section{The problem}

Rappold et al. (1984) have investigated the distribution of distance between specific regions of the sex chromosomes in male and female human lymphocyte nuclei by in situ hybridization experiments with two chromosome specific DNA probes CY1 and pXBR (see above). In this appendix three distinct model distributions of distances will be considered:

(a) The labelled regions of both chromosomes lie in the interior of a cell nucleus;

(b) One labelled region lies in the interior and the other at the boundary of the nucleus;

(c) Both labelled regions lie at the boundary of the nucleus.

Each model is based on the following assumptions:

(i) The cell nucleus of human lymphocytes can be considered as the interior of a sphere;

(ii) The position of the labelled region of one chromosome either in the interior of the cell nucleus (region 1) or at its boundary (region 2) is random and independent of the position of the other labelled chromosome segment; (iii) Microphotographs used for measurements of distances represent an orthogonal projection of the cell nucleus onto a plane.

\section{The mathematical model}

Consider a pair of independently and uniformly distributed random points, $P_{1}$ and $P_{2}$, of which $i$ are in the interior and $j$ are on the boundary (surface) of a sphere of radius $R(0 \leq i, j \leq 2$, $i+j=2$ ), i.e., for each of the two points the probability that it lies in any region of the interior of the sphere (resp. of its surface) shall be proportional to the volume (resp. area) of this region, independently from the position of the other point. Let $r(0 \leq r \leq 2 R)$ be the distance between $P_{1}$ and $P_{2}$ and $f_{i, j}(r)$ the probability density function (p.d.f.) of $r$. Then it is well known that:
(1a) $f_{2,0}(r)=\frac{3}{R}\left(\frac{r}{R}\right)^{2}\left[1-\frac{3}{4}\left(\frac{r}{R}\right)+\frac{1}{16}\left(\frac{r}{R}\right)^{3}\right]$,
(1b) $f_{1,1}(r)=\frac{3}{4 R}\left(\frac{r}{R}\right)^{2} \cdot\left(2-\frac{r}{R}\right)$, 
(1c) $f_{0,2}(r)=\frac{1}{2 R}\left(\frac{r}{R}\right)$

[for (1a) see, e.g., Hammersley 1950, for (1b) and (1c) see Miles 1971].

In order to obtain the distribution of the distance $b$ between the images of $P_{1}$ and $P_{2}$ by orthogonal projection onto a plane, which apparently has not been derived up to now, one has first to go back to the distribution of the vector $\mathfrak{r}=\mathfrak{r}_{1}-\mathfrak{r}_{2}$, where $\mathfrak{r}_{1}$ and $\mathfrak{r}_{2}$ are the radius vectors from the centre of sphere to $P_{1}$ and $P_{2}$, respectively. Because of the spherical symmetry of the model, the distribution of $r$ depends only on $r=|\mathfrak{r}|(|\mathfrak{r}|=$ length of $\mathfrak{r}$ ), and as the set of all vectors with fixed length $r$ drawn from the same origin describes a sphere of radius $r$, the p.d.f. of the vector $\mathfrak{r}$ is:

$$
f_{i, j}^{*}(\mathfrak{r})=\frac{1}{4 \pi r^{2}} f_{i, j}(r)
$$

We now decompose $r$ into the sum of a vector $\mathfrak{b}$ situated in the plane of projection ("projection vector") and a vector 3 perpendicular to this plane. To any vector $\mathfrak{r}$ with the same projection vector $b$ there belongs exactly one vector $z$ with

$$
\mathfrak{z}=z \mathfrak{z}_{0},-\sqrt{4 R^{2}-b^{2}} \leq z \leq \sqrt{4 R^{2}-b^{2}}
$$

and inversely, where $3_{0}$ is a constant unit vector and $b=|\mathbf{b}|$ (cf. Fig. 1). To obtain the p.d.f. of $\mathfrak{b}$ one has to integrate $f_{i j}^{*}(\mathfrak{r})$ over $z$ :

$$
g_{i, j}^{*}(\mathfrak{b})=\frac{1}{2 \pi} \int_{0}^{\sqrt{4 R^{2}-b^{2}}}\left[f_{i, j}\left(\sqrt{z^{2}+b^{2}}\right) /\left(z^{2}+b^{2}\right)\right] d z
$$

Finally the p.d.f. of $b$ is obtained by integrating (3) over all vectors $b$ with the same length $b$

$$
g_{i, j}(b)=b \int_{0}^{\sqrt{4 R^{2}-b^{2}}}\left[f_{i, j}\left(\sqrt{\left.z^{2}+b^{2}\right)} /\left(z^{2}+b^{2}\right)\right] d z\right.
$$

For simplification we introduce $b=2 R \beta$ in the following derivations.

Case $a:(i, j)=(2,0)$, i.e., both points $P_{1}$ and $P_{2}$ lie in the interior of the sphere.

From (1a) and (4), using the integral formulas

$$
\begin{aligned}
\int \sqrt{z^{2}+\beta^{2}} d z= & \frac{z}{2} \sqrt{z^{2}+\beta^{2}}+\frac{\beta^{2}}{2} \ln \left(z+\sqrt{z^{2}+\beta^{2}}\right) \\
& + \text { const, }
\end{aligned}
$$

$$
\begin{aligned}
\int z^{2} \sqrt{z^{2}+\beta^{2}} d z= & \frac{z}{8}\left(2 z^{2}+\beta^{2}\right) \sqrt{z^{2}+\beta^{2}} \\
& -\frac{\beta^{2}}{8} \ln \left(z+\sqrt{z^{2}+\beta^{2}}\right)+\text { const }
\end{aligned}
$$

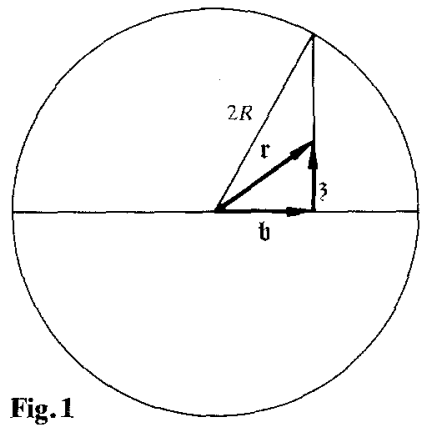

(see, e.g., Gröbner and Hofreiter 1961), we get

$$
\begin{aligned}
g_{2,0}(b) & =\frac{3 b}{2 R^{2}} \int_{0}^{\sqrt{4 R^{2}-b^{2}}}\left[2-\frac{3}{2 R}\left(z^{2}+b^{2}\right)^{\frac{1}{2}}+\frac{1}{8 R^{3}}\left(z^{2}+b^{2}\right)^{\frac{3}{2}}\right] d z \\
& =\frac{6 \beta}{R} \int_{0}^{\sqrt{1-\beta^{2}}}\left[2+\left(\beta^{2}-3\right) \sqrt{z^{2}+\beta^{2}}+z^{2} \sqrt{z^{2}+\beta^{2}}\right] d z \\
& =\frac{9 \beta}{4 R}\left[\left(\beta^{2}+2\right) \sqrt{1-\beta^{2}}+\beta^{2}\left(\beta^{2}-4\right) \ln \frac{1+\sqrt{1-\beta^{2}}}{\beta}\right]
\end{aligned}
$$

Using the likewise well-known integral formulas

$$
\begin{array}{r}
\int x^{k} \sqrt{1-x^{2}} d x=\frac{1}{k+1} \sin ^{k+1} \varphi \cos \varphi+\frac{1}{k+1} \int \sin ^{k+2} \varphi d \varphi \\
(k=0,1,2, \ldots)
\end{array}
$$

$$
\begin{aligned}
\int x^{k} \ln \frac{1+\sqrt{1-x^{2}}}{x} d x= & \frac{x^{k+1}}{k+1} \ln \frac{1+\sqrt{1-x^{2}}}{x} \\
& +\frac{1}{k+1} \int \sin ^{k} \varphi d \varphi \quad(k=0,1,2, \ldots)
\end{aligned}
$$

(with $\sin \varphi=x$ ), and

$$
\begin{array}{r}
\int \sin ^{2 k+1} \varphi d \varphi=\sum_{v=0}^{k}(-1)^{v+1}\left(\begin{array}{l}
k \\
v
\end{array}\right) \frac{1}{2 v+1} \cos ^{2 v+1} \varphi \\
(k=0,1,2, \ldots)
\end{array}
$$

we obtain the distribution function of $b$ by integration of $g_{2,0}(b)$ :

$$
\begin{aligned}
G_{2,0}(b)= & \int_{0}^{b} g_{2,0}(x) d x=2 R \int_{0}^{\beta} g_{2,0}(2 R t) d t \\
= & 1+\frac{1}{4}\left(3 \beta^{4}+16 \beta^{2}-4\right) \sqrt{1-\beta^{2}} \\
& +\frac{3}{4} \beta^{4}\left(\beta^{2}-6\right) \ln \frac{1+\sqrt{1-\beta^{2}}}{\beta}
\end{aligned}
$$

In the same way the moments of the distribution of $b$ can be obtained:

$$
\begin{aligned}
m_{k}= & \int_{0}^{2 R} x^{k} g_{2,0}(x) d x=(2 R)^{k} \int_{0}^{1} t^{k} g_{2,0}(2 R t) d t \\
= & 9(2 R)^{k}\left[\frac{k+5}{(k+4)(k+6)} \int_{0}^{\pi / 2} \sin ^{k+5} \varphi d \varphi\right. \\
& \left.-\frac{k}{(k+2)(k+4)} \int_{0}^{\pi / 2} \sin ^{k+3} \varphi d \varphi\right]
\end{aligned}
$$

and hence, using the product formula of Wallis

$$
\begin{array}{r}
\text { (12a) } m_{2 s}=(2 R)^{2 s} \frac{9}{(s+1)(s+2)(2+3)} \prod_{v=1}^{s+1} \frac{2 v}{2 v+1}(s=1,2, \ldots), \\
\text { (12b) } m_{2 s+1}=(2 R)^{2 s+1} \frac{36 \pi}{(2 s+4)(2 s+5)(2 s+7)} \prod_{v=1}^{s+1} \frac{2 v-1}{2 v} \\
(s=0,1,2, \ldots)
\end{array}
$$

Especially:

$$
\begin{aligned}
E(b) & =m_{1}=2 R \frac{9 \pi}{70}=0.403919(2 R), \\
\operatorname{var}(b) & =m_{2}-m_{1}^{2}=(2 R)^{2}\left[\frac{1}{5}-\left(\frac{9 \pi}{70}\right)^{2}\right] \\
& =0.036849(2 R)^{2} .
\end{aligned}
$$


Case $b:(i, j)=(1,1)$, i.e., $P_{1}$ lies in the interior and $P_{2}$ on the surface of the sphere.

From (1b) and (4), again using (5) and (6), we get

$$
\begin{aligned}
g_{1,1}(b) & =\frac{6 \beta}{R} \int_{0}^{\sqrt{1-\beta^{2}}}\left(1-\sqrt{z^{2}+\beta^{2}}\right) d z \\
& =\frac{3 \beta}{R}\left(\sqrt{1-\beta^{2}}-\beta^{2} \ln \frac{1+\sqrt{1-\beta^{2}}}{\beta}\right.
\end{aligned}
$$

Hence, using $(8)-(10)$,

$$
\begin{aligned}
G_{1,1}(b) & =\int_{0}^{b} g_{1,1}(x) d x= \\
& =1+\frac{1}{2}\left(5 \beta^{2}-2\right) \sqrt{1-\beta^{2}}-\frac{3}{2} \beta^{4} \ln \frac{1+\sqrt{1-\beta^{2}}}{\beta}
\end{aligned}
$$

and

$$
m_{2 s}=(2 R)^{2 s} \frac{3}{(s+1)(s+2)} \prod_{v=1}^{s+1} \frac{2 v}{2 v+1} \quad(s=1,2, \ldots)
$$

(15b) $m_{2 s+1}=(2 R)^{2 s+1} \frac{6 \pi}{(2 s+3)(2 s+5)} \prod_{\nu=1}^{s+2} \frac{2 v-1}{2 v}$

$$
(s=0,1,2, \ldots)
$$

Especially:

$$
\begin{aligned}
& \begin{aligned}
E(b)= & m_{1}=2 R \frac{3 \pi}{20}=0.471239(2 R), \\
\operatorname{var}(b) & =m_{2}-m_{1}^{2}=(2 R)^{2}\left[\frac{4}{15}-\left(\frac{3 \pi}{20}\right)^{2}\right] \\
& =0.044601(2 R)^{2} .
\end{aligned}
\end{aligned}
$$

Case c: $(i, j)=(0,2)$, i.e., both points $P_{1}$ and $P_{2}$ are situated on the surface of the sphere.

From (1c) and (4), using the integral formula

$$
\int \frac{d z}{\sqrt{z^{2}+\beta^{2}}}=\ln \left(z+\sqrt{z^{2}+\beta^{2}}\right)+\text { const }
$$

we obtain

$$
g_{0,2}(b)=\frac{\beta}{R} \int_{0}^{\sqrt{1-\beta^{2}}} \frac{d z}{\sqrt{z^{2}+\beta^{2}}}=\frac{\beta}{R} \ln \frac{1+\sqrt{1-\beta^{2}}}{\beta} .
$$

Hence, using (9) and (10),

$$
G_{0,2}(b)=\int_{0}^{b} g_{0,2}(x) d x=1-\sqrt{1-\beta^{2}}+\beta^{2} \ln \frac{1+\sqrt{1-\beta^{2}}}{\beta}
$$

and

(19a) $m_{2 s}=(2 R)^{2 s} \frac{1}{s+1} \prod_{v=1}^{s} \frac{2 v}{2 v+1} \quad(s=1,2, \ldots)$,

(19b) $m_{2 s+1}=(2 R)^{2 s+1} \frac{\pi}{2 s+3} \prod_{v=1}^{s+1} \frac{2 v-1}{2} \quad(s=0,1,2, \ldots)$

Especially:

$$
\begin{aligned}
& E(b)=m_{1}=2 R \frac{\pi}{6}=0.523599(2 R), \\
& \operatorname{var}(b)=m_{2}-m_{1}^{2}=(2 R)^{2}\left[\frac{1}{3}-\left(\frac{\pi}{6}\right)^{2}\right] \\
& =0.059178(2 R)^{2} .
\end{aligned}
$$

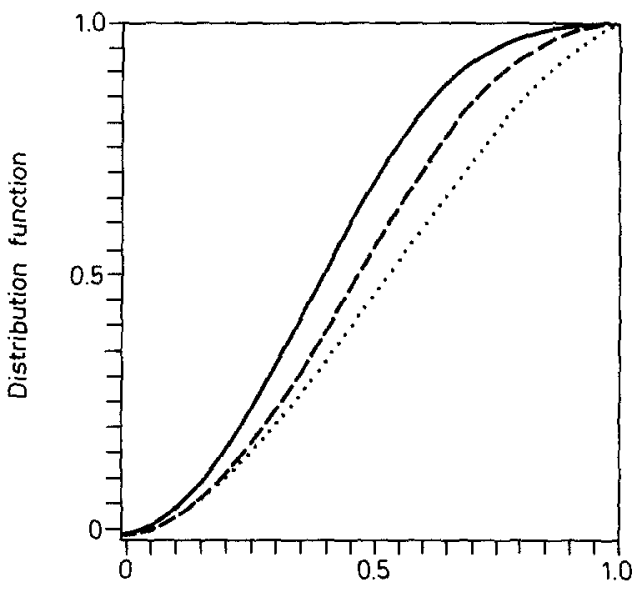

Distance/diameter

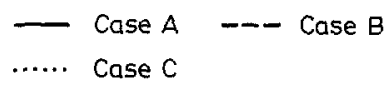

Fig. 2. Distribution function of distance $b$ between the projections of two random points

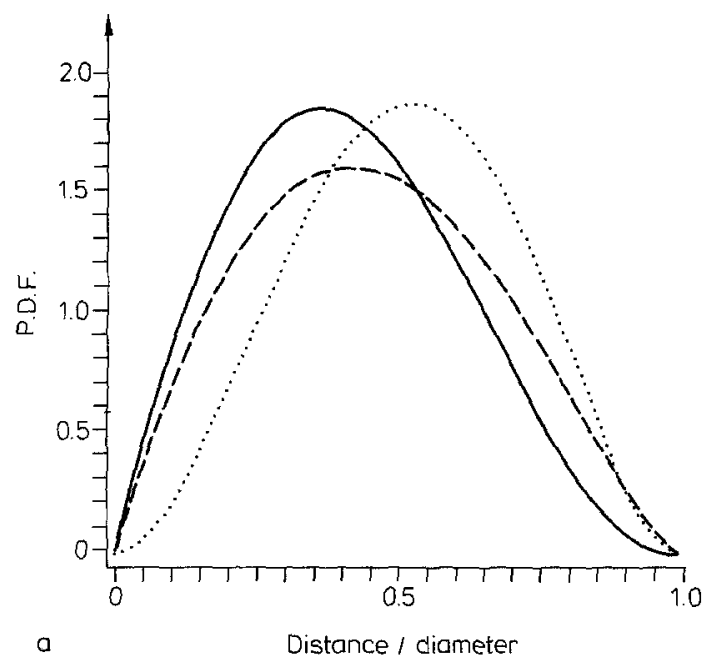

Fig.3a. Comparison of probability density functions (p.d.f.) for the distributions of three distances in case a: ——Distance between the projections of two random points in the sphere onto a plane. .......... Distance between two random points in the sphere themselves. - - - Distance between two random points in the circle

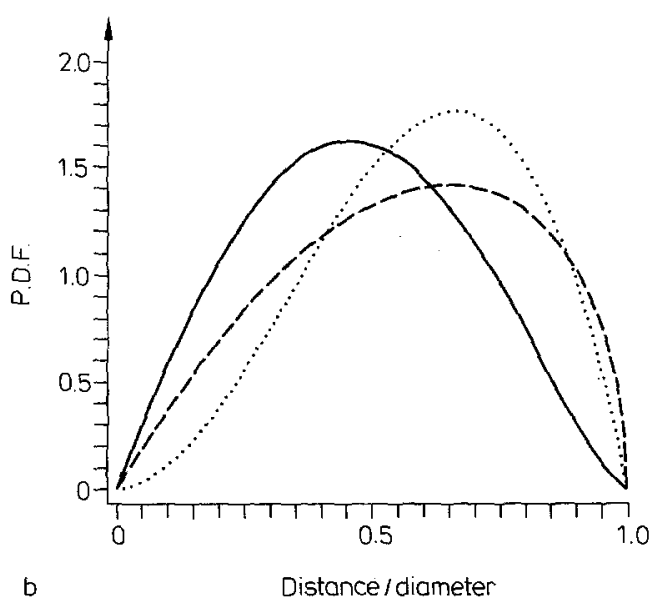

Fig.3b. Comparison of the three p.d.f.'s in case b, for explanations of the curves cf. Fig. 3 a 


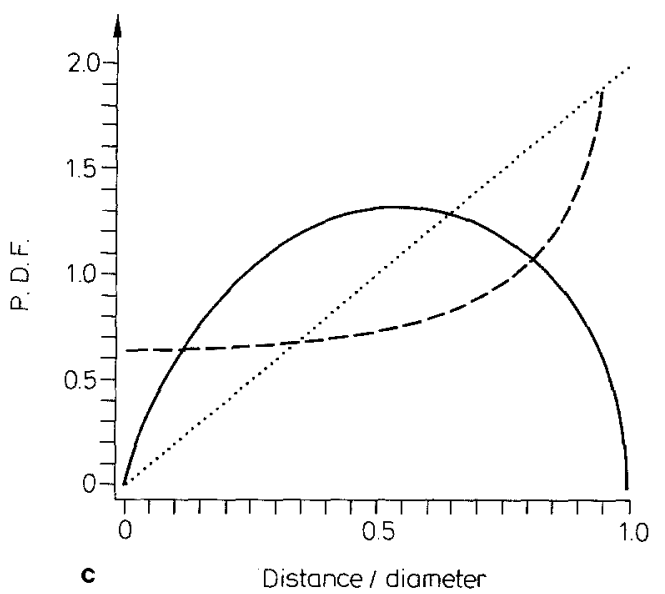

Fig.3c. Comparison of the three p.d.f.'s in case c, for explanation of the curves cf. Fig. 3 a

Figure 2 shows the distribution functions of $b$ for the three cases a-c together in one graphical representation. In Fig. $3 a-c$, the p.d.f. of $b$ in each of the three cases is compared with the corresponding p.d.f. $f_{i, j}(r)$ of the distance between the points $P_{1}$ and $P_{2}$ themselves and with the corresponding p.d.f. $h_{i, j}(r)$ of distance for the analogous model in the plane: two random points of which $i$ are in the interior and $j$ are on the boundary of a circle of radius $R(0 \leq i, j \leq 2, i+j=2)$. The latter p.d.f. is given by the formulae (see Miles 1971):

(20a) $h_{2,0}(r)=\frac{8 \beta}{\pi R}\left(\operatorname{arccoss} \beta-\beta \sqrt{1-\beta^{2}}\right)$,

(20b) $h_{1,1}(r)=\frac{4 \beta}{\pi R} \arccos \beta$,

(20c) $h_{0,2}(r)=\frac{1}{\pi R} \frac{1}{\sqrt{1-\beta^{2}}}$,

were again $\beta=r / 2 R$.

\section{References}

Gröbner W, Hofreiter N (1961) Integraltafel, Teil 1: Unbestimmte Integrale, 3. Aufl. Springer, Wien

Hammersley JM (1950) The distribution of distance in a hypersphere. Ann Math Stat 21:447-452

Miles RE (1971) Isotropic random simplices. Adv Appl Probability $3: 353-382$

Received March 28 / Revised April 10, 1984 